\title{
Aplicação de caracteres morfoanatômicos foliares na taxonomia de Campomanesia Ruiz \& Pavón (Myrtaceae) ${ }^{1}$
}

\begin{abstract}
Marla Ibrahim Uehbe de Oliveira ${ }^{2,4}$, Ligia Silveira Funch ${ }^{2}$, Francisco de Assis Ribeiro dos Santos ${ }^{2}$ Leslie Roger Landrum $^{3}$
Recebido em 23/03/2011. Aceito em 16/05/2011

RESUMO

(Aplicação de caracteres morfoanatômicos foliares na taxonomia de Campomanesia Ruiz \& Pavón (Myrtaceae)). Para avaliar a utilidade de caracteres morfoanatômicos na identificação de materiais botânicos de Campomanesia Ruiz \& Pavón, especialmente aqueles estéreis, o padrão de nervação foliar e a configuração do arco vascular no pecíolo de 15 táxons presentes no estado da Bahia foram analisados. O padrão de nervação secundário camptódromo-broquidódromo mostrou-se uniforme entre os táxons, assim como a nervação última marginal em arcos e a nervação terciária do tipo reticulada ortogonal. Diferenças foram observadas quando considerados a presença de nervuras intersecundárias e o tipo de aréolas na lâmina foliar, e a configuração do arco vascular e a natureza da bainha perivascular no pecíolo, podendo estes caracteres ser utilizados para fins taxonômicos.
\end{abstract}

Palavras-chave: nervação, anatomia foliar, taxonomia, Myrtaceae, Bahia

\begin{abstract}
(Application of morpho-anatomical leaf characters in Campomanesia Ruiz \& Pavón (Myrtaceae) taxonomy). To evaluate the utility of morpho-anatomical leaf characters in the identification of botanic material of Campomanesia Ruiz \& Pavón, especially when sterile, leaf venation pattern and shape of the vascular arch in the petiole of 15 taxa in Bahia state were analysed. The camptodromous-brochydodromous secondary venation pattern was uniform among the taxa, as well as the ultimate marginal venation in arches and orthogonal reticulate tertiary venation. Differences were observed in the presence of intersecondary veins and areolation in the leaf blade, and shape of the vascular arch and nature of the vascular bundle sheaths in the petiole, characters that could be used for taxonomic purposes.
\end{abstract}

Key words: venation, leaf anatomy, taxonomy, Myrtaceae, Bahia

\section{Introdução}

No Brasil, todas as Myrtaceae estão circunscritas na tribo Myrteae, tradicionalmente divida em Myrciinae, Eugeniinae e Myrtinae (McVaugh 1968; Barroso et al. 1991; Landrum \& Kawasaki 1997), subtribos que não se sustentam como monofiléticas (Wilson et al. 2001; Wilson et al. 2005; Lucas et al. 2005). Estudos recentes identificaram sete grupos informais na tribo e, apesar de serem separados tanto por dados moleculares quanto por uma combinação de caracteres morfológicos, são pouco sustentados e necessitam de uma amostragem mais ampla para que haja uma reclassificação em níveis inferiores dentro da mesma (Lucas et al. 2007).

Como parte da tradicional subtribo Myrtinae e do informal “Grupo Pimenta” (Lucas et al. 2007) Campomanesia
Ruiz \& Pavón possui cerca de 40 espécies (Govaerts et al. 2008; Landrum \& Oliveira 2010; Proença et al. 2010; Proença et al.2011) distribuídas nas regiões tropical e subtropical da América do Sul, ocorrendo em diversos ambientes, como matas, cerrados, campos e restingas (Landrum 1986; Landrum \& Kawasaki 1997).

De acordo com Landrum (1986), as espécies de Campomanesia são arbustos ou árvores que se separam de outras Myrtaceae pelo número de lóculos no ovário, (3-)4-18, pela presença de uma parede glandular protetora, funcionando como uma falsa testa na semente, e aborto de todos ou quase todos os óvulos, exceto um em cada lóculo. A inflorescência pode ser uniflora ou um dicásio com até três flores, existindo algumas exceções. Os frutos, referidos por "guabiroba", são apreciados localmente e cultivados em pequena escala, em-

\footnotetext{
1 Parte da dissertação do primeiro Autor

2 Universidade Estadual de Feira de Santana, Programa de Pós-Graduação em Botânica, Feira de Santana, BA, Brasil

3 Arizona State University, School of Life Sciences, Tempe, AZ, USA

4 Autor para correspondência: marlauehbe@yahoo.com.br
} 
bora possuam considerável potencial econômico (Legrand \& Klein 1977; Landrum 1986; Carrara 1997; Kawasaki \& Landrum 1997; Barroso et al. 2004; Lorenzi et al. 2006).

Apesar de as espécies de Campomanesia serem confundidas com as de Psidium L. (McVaugh 1968), resultados preliminares sobre a filogenia do gênero obtidos através da análise de seqüências nucleares, como ITS e ETS, sustentamno como monofilético (I.R. Costa, com. pess.), confirmando a idéia de este ser um dos gêneros mais bem definidos dentro da família (McVaugh 1968; Landrum 1986). No entanto, os limites entre algumas espécies são pouco compreendidos e, por esta razão, Landrum (1986) citou a formação de três grupos informais baseados principalmente em caracteres florais: "Complexo Campomanesia guazumifolia" [C. guazumifolia (Cambess.) O. Berg, C. schlechtendaliana (O. Berg) Nied., C. rufa (O. Berg) Nied. e C. sessiliflora(O. Berg) Mattos], "Complexo C. grandiflora" [C. grandiflora (Aubl.) Sagot, C. neriiflora (O. Berg) Nied., C. guaviroba (DC.) Kiaersk., C. speciosa (Diels) McVaugh e C. lineatifolia Ruiz \& Pav.] e "Complexo C. xanthocarpa" [C. xanthocarpa O. Berg, C. aromatica (Aubl.) Griseb., C. adamantium (Cambess.) O. Berg, C. reitziana Legrand, C. velutina (Cambess.) O. Berg, C. eugenioides (Cambess.) Legrand, C. pubescens (DC.) O. Berg, C. pabstiana Mattos \& Legrand e C. aurea O. Berg].

Trabalhos envolvendo outras abordagens, como anatomia, podem colaborar no entendimento de tais limites. A arquitetura foliar, no que se refere aos padrões de nervação, a configuração marginal, a forma da folha e a posição de glândulas, constitui-se em um aspecto consistente no reconhecimento de plantas em nível de espécie (Hickey 1973; 1979). Em Myrtaceae, podem ser citados os estudos morfoanatômicos conduzidos por Du Plessis \& Van Wik (1982), Carr et al. (1986), Fontenelle et al. (1993), Fontenelle et al. (1994), Costa et al. (1995), Carrara (1997), Gomes \& Neves (1997), Donato \& Morretes (2007), e Gomes et al. (2009). Klucking (1988) descreveu os padrões de nervação foliar na família e citou particularidades para Campomanesia também observadas por Cardoso \& Sajo (2006) quando estes estudaram espécies pertencentes às tradicionais subtribos de Myrteae. Da mesma forma, o posicionamento dos feixes vasculares no pecíolo constitui um caráter relevante no reconhecimento de grupos (Howard 1979), como constatado por Cardoso \& Sajo (2004) em Eugenia L., possibilitando a identificação de materiais estéreis.

Assim, este trabalho buscou diferenças siginificativas entre os padrões de nervação foliar e a configuração do arco vascular no pecíolo dos táxons de Campomanesia presentes no estado da Bahia, com o intuito de conhecer esses padrões e avaliar a utilidade dos mesmos em estudos taxonômicos no grupo, especialmente na identificação de materiais estéreis.

\section{Material e métodos}

Os materiais utilizados neste trabalho correspondem a 15 táxons de Campomanesia registrados no estado da Bahia por Oliveira et al. (no prelo) . As amostras obtidas em campo apresentam vouchers no herbário HUEFS e as demais foram retiradas de materiais herborizados depositados nos herbários ALCB, ASU, HUEFS, NY e R (Tab. 1, siglas segundo Holmgren et al. 1990). Salienta-se que amostras de Campomanesia blanchetiana Landrum \& Ibrahim e de C. sessiliflora var. bullata (Barb. Rodr.) Landrum coletadas no estado não se encontraram disponíveis durante o estudo, tornando-se necessária a utilização de materiais provenientes de outras localidades no caso deste último táxon.

Folhas perfeitamente desenvolvidas do terceiro e quarto nós foram selecionadas para o estudo, sendo materiais frescos acondicionados em $\mathrm{FAA}_{70}$ por $48 \mathrm{~h}$ e transferidos para etanol 70\% (Kraus \& Arduin 1997). O material herborizado passou por um tratamento de reidratação com água e glicerina $50 \%$.

Para a observação do padrão de nervação, foram diafanizadas folhas adotando-se a técnica de Shobe \& Lersten (1967) com modificações, como a substituição do cloral hidratado por hipoclorito de sódio. Após este processo e posterior coloração com safranina, as folhas foram montadas em lâminas de vidro temporárias e fotografadas com auxílio de negatoscópio e de câmera digital SONY Cyber Shot modelo DSC-S650 com resolução de 7.2 MP. Para a análise da rede menor de nervação, fragmentos do terço mediano das folhas foram montados em lâminas semipermanentes e fotografados em estereomicroscópio Zeiss Stemi SV6 com auxílio do programa AxioVision 3.1 Zeiss. A descrição e análise dos materiais seguiram os tipos básicos de Hickey $(1973 ; 1979)$.

Com relação à análise da configuração do arco vascular e da natureza das células da bainha perivascular no pecíolo, cortes transversais à mão livre foram realizados na região mediana do mesmo. Em seguida, os cortes foram clarificados, corados com safrablau, e montados em lâminas semipermanentes com glicerina. As fotomicrografias foram realizadas em microscópio de luz Zeiss Hal 100 Axioskop com auxílio de camêra digital Olympus modelo C-5060 com resolução de 5.1 MP, e os tipos foram descritos de acordo com Howard (1979).

\section{Resultados e discussão}

As folhas de Campomanesia Ruiz \& Pavón analisadas possuem formas diversas, sendo elípticas ou ovadas em sua maioria. Os ápices vão de agudos a acuminados e as bases de agudas a cordadas, estando estas presentes em C. dichotoma e C. eugenioides var. desertorum apenas. As margens são inteiras, revolutas, crenadas, crenuladas ou onduladas. Klucking (1988) considerou a ocorrência de margens não-inteiras, algo incomum em Myrtaceae, uma característica diferencial para o gênero, citando margens remotamente denteadas que se apresentam onduladas em $C$. aromatica e C. guazumifolia, o que também foi constatado neste estudo para C. eugenioides var. desertorum (Fig. 4) e 
Tabela 1. Lista de espécimes de Campomanesia Ruiz \& Pavón analisados. $\left({ }^{*}\right)$ representando os materiais frescos obtidos em campo, Bahia.

\begin{tabular}{|c|c|c|}
\hline Táxons & Coletor, número e herbário & Local e tipo vegetacional \\
\hline Campomanesia anemonea Landrum & W.W.Thomas et al. 10251 (ASU) & Itapetinga (floresta atlântica) \\
\hline C. aromatica (Aubl.) Griseb. & M. Ibrahim 140 (HUEFS) * & Feira de Santana (caatinga) \\
\hline \multirow[t]{3}{*}{ C. dichotoma (O. Berg) Mattos } & E.P.Queiroz 836 (HUEFS) & Mata de São João (restinga) \\
\hline & L.N.Silva et al. 46 (HUEFS) & Conde (restinga) \\
\hline & M. Ibrahim 123 (HUEFS) * & Camaçari (restinga) \\
\hline \multirow[t]{3}{*}{ C. eugenioides var. desertorum (DC.) Landrum } & A.O.Moraes et al. 158 (HUEFS) & Itatim (caatinga) \\
\hline & E. Melo 5694 (HUEFS)* & Feira de Santana (caatinga) \\
\hline & F. França et al. 5433 (HUEFS) & Campo Formoso (caatinga/cerrado) \\
\hline C. grandiflora (Aubl.) Sagot & Pimenta Vellozo 957 (R) & Ilhéus (restinga) \\
\hline C. guaviroba (DC.) Kiaersk. & E.P.Queiroz 1129 (ALCB) & Mata de São João (restinga) \\
\hline \multirow[t]{2}{*}{ C. guazumifolia (Cambess.) O. Berg } & S.A.Mori et al. $10666(\mathrm{NY})$ & Prado (restinga) \\
\hline & W.W.Thomas et al. $7202(\mathrm{NY})$ & Uruçuca (floresta atlântica) \\
\hline \multirow[t]{3}{*}{ C. ilhoensis Mattos } & A.Popovkin $255(\mathrm{HUEFS})^{*}$ & Entre Rios (floresta atlântica) \\
\hline & M.Ibrahim et al. 122 (HUEFS)* & Alagoinhas (floresta altântica) \\
\hline & N.G.Jesus et al. 510 (HUEFS) & Alagoinhas (floresta altântica) \\
\hline \multirow[t]{3}{*}{ C. laurifolia Gardner } & M.M.Lopes et al. 1010 (BHCB) & Arataca (floresta atlântica) \\
\hline & P.Gasson et al. 5976 (HUEFS) & Morro do Chapéu (campo rupestre) \\
\hline & R.A.X.Borges et al. 684 (HUEFS) & Camacan (floresta atlântica) \\
\hline C. schlechtendaliana (O. Berg) Nied. & G.Hatschbach et al. 71569 (HUEFS) & Espírito Santo, Itapemirim (restinga) \\
\hline \multirow{2}{*}{ C. sessiliflora var. sessiliflora (O. Berg) Mattos } & G.Hatschbach et al. 7569 (HUEFS) & Milagres (floresta) \\
\hline & M.L.Guedes 2976 (ALCB) & Caetité (campos gerais) \\
\hline C. sessiliflora var. bullata (Barb. Rodr.) Landrum & E.Zardini et al. 48331 (ASU) & Paraguai, San Pedro (cerrado) \\
\hline \multirow[t]{3}{*}{ C. sessiliflora var. lanuginosa (Barb. Rodr. ex Chodat \& Hassl.) Landrum } & L.P. de Queiroz et al. 4011 (HUEFS) & Morro do Chapéu (cerrado) \\
\hline & M.Ibrahim et al. 35 (HUEFS) * & Mucugê (campo rupestre) \\
\hline & M.Ibrahim et al. 132 (HUEFS) * & Rio de Contas (cerrado) \\
\hline \multirow[t]{2}{*}{ C. velutina (Cambess.) O. Berg } & R.P.Orlandi 302 (HUEFS) & Morro do Chapéu (caatinga) \\
\hline & W.Ganev 1511 (HUEFS) & Abaíra (carrasco) \\
\hline \multirow[t]{2}{*}{ C. xanthocarpa O. Berg } & E.Woodgyer et al. 2391 (HUEFS) & Morro do Chapéu (cerrado) \\
\hline & R.M.Harley et al. 6088 (HUEFS) & Morro do Chapéu (campo rupestre) \\
\hline
\end{tabular}

C. schlechtendaliana (Fig. 10). Os tricomas são simples e podem estar presentes em ambas as faces da lâmina foliar, como em C. sessiliflora var. lanuginosa e C. velutina; ao longo das nervuras primárias e secundárias, como em $C$. laurifolia e C. eugenioides var. desertorum; e na axila das nervuras secundárias com a principal formando tufos, como em C. guaviroba. Além disso, também podem ser encontrados pontos translúcidos e pretos, que representam, respectivamente, cavidades secretoras e glândulas laminares (Fig. 27-28), considerados típicos da família por Metcalfe \& Chalk (1950).

Em geral, as nervuras são impressas na face adaxial e proeminentes na face abaxial, apresentando-se mais marcadas naquelas buladas, como Campomanesia guazumifolia, C. schlechtendaliana, C. sessiliflora var. lanuginosa e $C$. xanthocarpa. A nervura primária é reta, com encurvamento próximo ao ápice, mais acentuado em $C$. anemonea, $C$. eugenioides var. desertorum, C. guaviroba, C. laurifolia, C. velutina e C. xanthocarpa.

As nervuras secundárias são curvadas, com espaçamento largo na maioria dos táxons, e possuem número de pares variando entre 5-15. Estas nervuras se anastomosam desde a base da folha, compondo arcos próximos à margem, sem formação de nervura intramarginal, uma das características do gênero (Landrum 1986; Klucking 1988). Desta forma, o padrão de nervação em todas as espécies estudadas é camptódromo-broquidódromo (Fig. 1-15), assim como constatado por Carrara (1997) para as espécies de Campomanesia do Rio de Janeiro. Este é também um tipo bastante comum em Eugenia L. (Costa et al. 1995; Cardoso \& Sajo 2004) e nas Myrtaceae brasileiras (Cardoso \& Sajo 2006), ainda que Klucking (1988) tenha assinalado o tipo acródromo como característico da família. Landrum (1986) comentou a ocorrência em Campomanesia laurifolia 


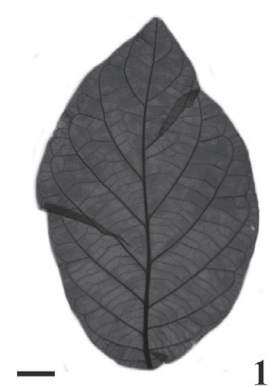

1

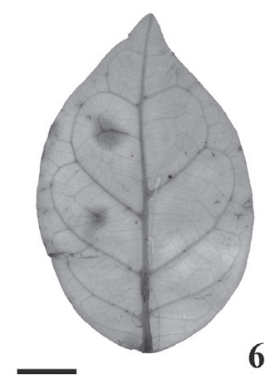

6

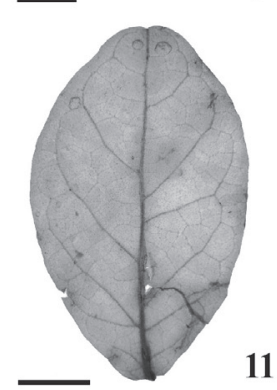

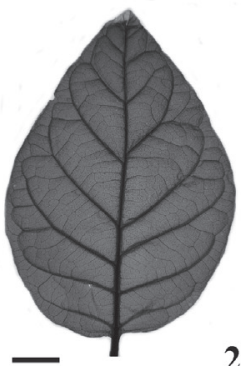
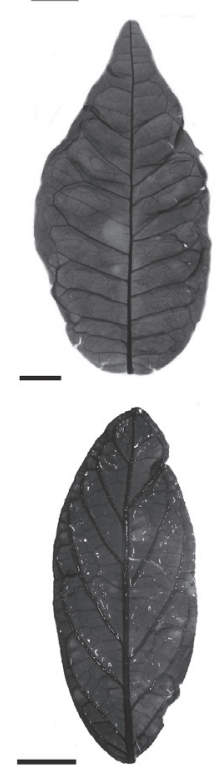

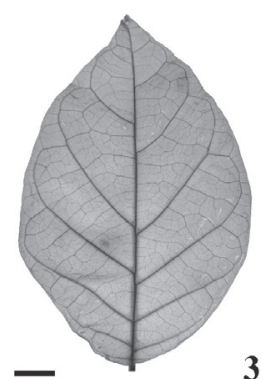

3

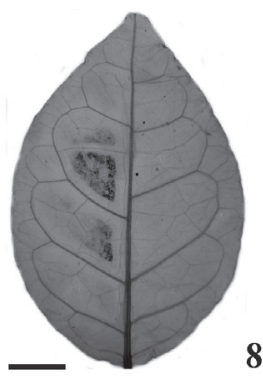

8

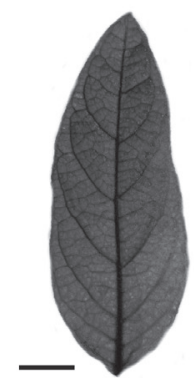

13

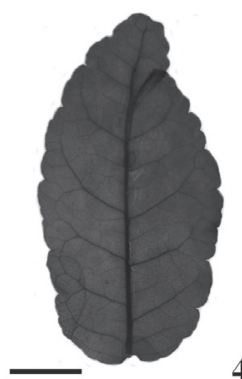

4
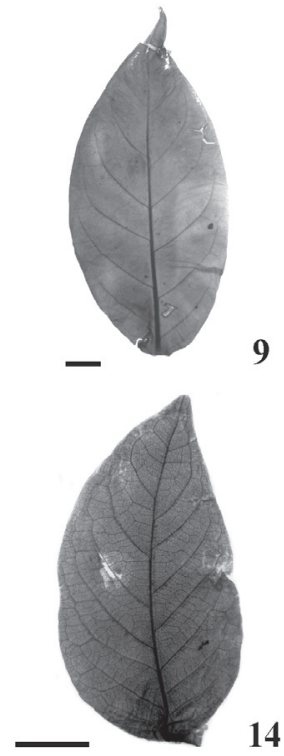

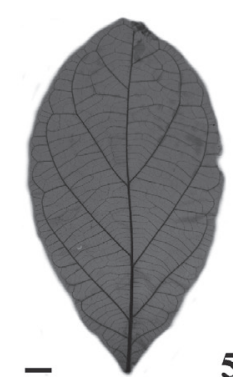

5

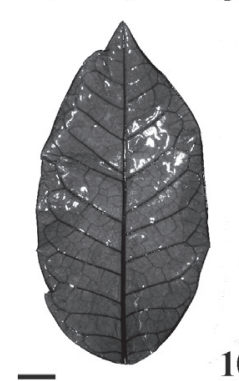

10

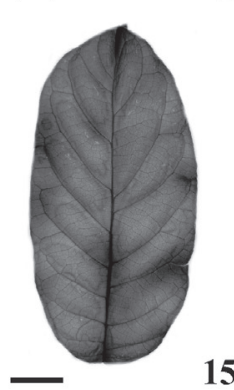

Figuras 1-15. Padrão de nervação nos táxons de Campomanesia Ruiz \& Pavón presentes no estado da Bahia. 1. C. anemonea Landrum. 2. C. aromatica (Aubl.) Griseb. 3. C. dichotoma (O. Berg) Mattos. 4. C. eugenioides var. desertorum (DC.) Landrum. 5. C. grandiflora (Aubl.) Sagot. 6. C. guaviroba (DC.) Kiaersk. 7. C. guazumifolia (Cambess.) O. Berg. 8. C. ilhoensis Mattos. 9. C. laurifolia Gardner. 10. C. schlechtendaliana (O. Berg) Nied. 11. C. sessiliflora var. sessiliflora (O. Berg) Mattos. 12. C. sessiliflora var. bullata (Barb. Rodr.) Landrum. 13. C. sessiliflora var. lanuginosa (Barb. Rodr. ex Chodat \& Hassl.) Landrum. 14. C. velutina (Cambess.) O. Berg. 15. C. xanthocarpa O. Berg. Escalas $=1 \mathrm{~cm}$.

do tipo eucamptódromo, no qual as nervuras secundárias conectam-se através de uma série de vênulas, sem formação de arcos próximos à margem (Hickey 1973; 1979), tipo não observado nos representantes analisados deste táxon.

$\mathrm{O}$ ângulo de divergência entre a nervura primária e as secundárias variou de $30^{\circ}$ a $60^{\circ}$, tipo agudo, podendo este ser estreito, com ângulos menores que $45^{\circ}$, identificado para a maior parte dos táxons; ou moderado, com ângulos entre $45^{\circ}$ e $65^{\circ}$, presentes em Campomanesia anemonea, $C$. aromatica, C. grandiflora, C. sessiliflora var sessiliflora, C. sessiliflora var. bullata e C. sessiliflora var. lanuginosa. Considerando o terço superior das folhas, as nervuras secundárias apresentaram ângulos de divergência mais obtusos em $C$. anemonea, C. aromatica, C. dichotoma, C. eugenioides var. desertorum, C. guaviroba, C. guazumifolia, C. ilhoensis e C. schlechtendaliana (Fig. 1-4, 6-8, 10).

A nervação última marginal em forma de arcos aparece em todos os táxons analisados, como em C. grandiflora (Fig. 16), possuindo menor calibre quando mais próximos à margem. Essa característica já havia sido assinalada para espécies de Psidium L., Myrceugenia O. Berg, Marlierea
Cambess. e Eugenia por Cardoso \& Sajo (2006), não sendo portanto diagnóstica para o gênero.

Uma característica marcante, também observada por Klucking (1988) em espécies de Campomanesia, foi a presença de nervuras interssecundárias simples (Tab. 2, Fig. 17). São originadas na nervura primária, e seguem um curso geralmente paralelo às nervuras secundárias, apresentando calibre intermediário entre estas e as nervuras terciárias (Hickey 1973; 1979). Neste estudo, essas nervuras ocorreram em C. anemonea, C. eugenioides var. desertorum, C. guaviroba, C. guazumifolia, C. ilhoensis, C. sessiliflora var sessiliflora, C. sessiliflora var. bullata e C. sessiliflora var. lanuginosa, podendo estar relacionadas à distância entre nervuras secundárias. Carrara (1997) comentou a facilidade de observação de tais nervuras em C. guaviroba e C. lundiana (Kiaersk.) Mattos, sendo este último táxon endêmico do estado do Rio de Janeiro e não analisado neste estudo.

O padrão de ramificação das nervuras terciárias não variou, ocorrendo em todas as espécies o tipo reticulado ortogonal (Fig. 18-32), no qual as nervuras se conectam entre si ou com nervuras secundárias opostas em ângulos 

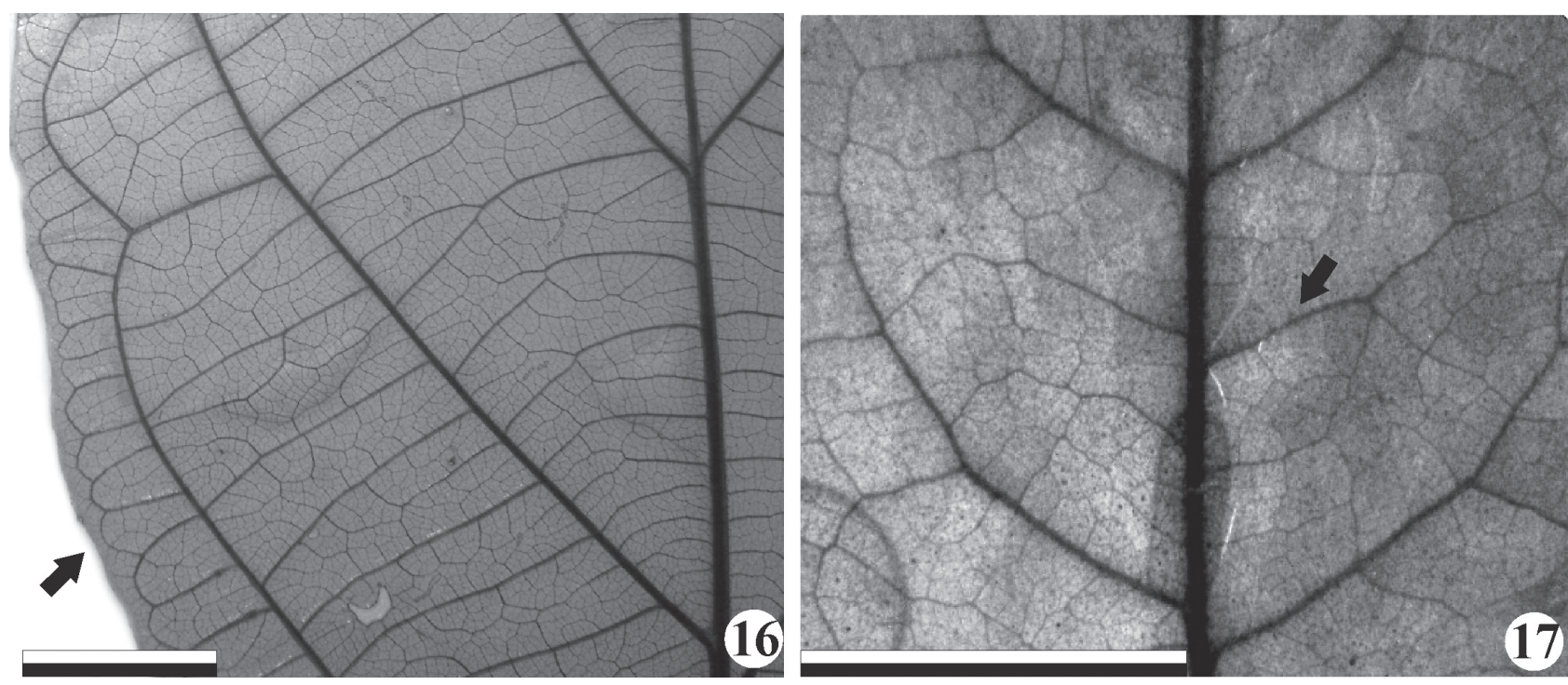

Figuras 16-17. Padrão de nervação nos táxons de Campomanesia Ruiz \& Pavón presentes no estado da Bahia. 16. Seta indicando nervação última marginal em arcos em C. grandiflora (Aubl.) Sagot. 17. Seta indicando presença de nervura interssecundária em C. eugenioides var. desertorum (DC.) Landrum. Escala = $1 \mathrm{~cm}$.

predominantemente retos. Nos estudos conduzidos por Cardoso \& Sajo (2006), somente o gênero Campomanesia apresentou este padrão, típico de algumas espécies da tradicional subtribo Myrtinae.

As menores áreas formadas por nervuras terciárias e nervuras de ordens menores originam as chamadas aréolas, que podem apresentar desenvolvimento, arranjo e forma diferentes, além de terem ou não vênulas livres em seu interior (Tab. 2, Fig. 33-47). São consideradas perfeitas quando possuem malha de tamanho e forma regulares (Fig. 33-36, 40, 44-45, 47); imperfeitas, quando as malhas variam em tamanho e se apresentam de maneira irregular (Fig. 37-39, 41-43, 46); ou incompletas, sem limitação dos lados da malha. Quando distribuídas de modo similar dentro de uma área em particular, caracterizam o arranjo orientado, tipo mais comum entre os táxons analisados (Fig. 33-36, 40, $44-45,47)$. Também revelam forma irregular a pentagonal, e as vênulas são simples ou ramificadas, estando ausentes em C. sessiliflora var. bullata, C. sessiliflora var. lanuginosa e C. xanthocarpa (Fig. 44-45, 47). Interessante notar que na variedade-tipo de C. sessiliflora as aréolas são características, com vênulas ramificadas de três vezes ou mais (Fig. 28, 43). Carrara (1997) observou tal ocorrência em ambas as variedades de C. schlechtendaliana, que neste estudo mostrou vênulas simples (Fig. 42).

A secção transversal do pecíolo na região mediana revelou contornos côncavo-convexos, com uma configuração do sistema vascular idêntica em todos os táxons. O feixe vascular apresentou-se em arco simples, aberto, indiviso e bicolateral, atributo observado em Eugenia (Costa et al. 1995; Cardoso \& Sajo 2004) e em outros gêneros da família (Metcalfe \& Chalk 1950). As extremidades variaram entre voltadas para o centro e fletidas (Fig. 48-62), sendo estas últimas representadas em Campomanesia guaviroba, $C$. guazumifolia, C. laurifolia e C. xanthocarpa (Fig. 53-54, 56, 62). Considerando a bainha perivascular, caráter significativo segundo Costa et al. (1995) e Cardoso \& Sajo (2004), somente C. ilhoensis e C. velutina apresentaram células de natureza parenquimática (Fig. 55-61), enquanto os demais táxons apresentaram-se constituídos por células de natureza mista (parenquimáticas e esclerenquimáticas).

Fibras esclerenquimáticas aparecem concentradas próximas às extremidades ou em toda a extensão da bainha. Ainda foram observados idioblastos, contendo cristais em forma de drusas, e cavidades secretoras (Fig. 63-64), cuja natureza do conteúdo não foi possível definir, apesar de ser relatada em outros gêneros a existência de óleos essenciais (Metcalfe \& Chalk 1950; Gomes \& Neves 1997; Donato \& Morretes 2005). Ambas as estruturas ocorreram conjuntamente e variaram em quantidade na região cortical ao redor do sistema vascular, não servindo como caracteres diferenciais entre os táxons.

Diante de tais informações, dentro dos complexos citados por Landrum (1986), Campomanesia grandiflora e $C$. guaviroba podem ser separadas pela ausência e presença de nervuras interssecundárias e de vênulas simples e ramificadas, respectivamente, além de apresentarem diferentes extremidades do arco vascular no pecíolo. Do mesmo modo, C. guazumifolia, C. schlechtendaliana e C. sessiliflora podem ser separadas, possuindo, ainda, desenvolvimento, arranjo e forma das aréolas diferentes. Entre as variedades desta última espécie, somente C. sessiliflora var. sessiliflora pode ser identificada por suas aréolas imperfeitas, arranjo ao acaso, formas irregulares e vênulas ramificadas. Já C. xanthocarpa, C. aromatica, C. engenioides var. desertorum e C. velutina apresentam diferenças no arranjo do arco vascular no pecíolo e na natureza das células da bainha perivascular. Apesar de não citada em nenhum complexo, $C$. ilhoensis possui 

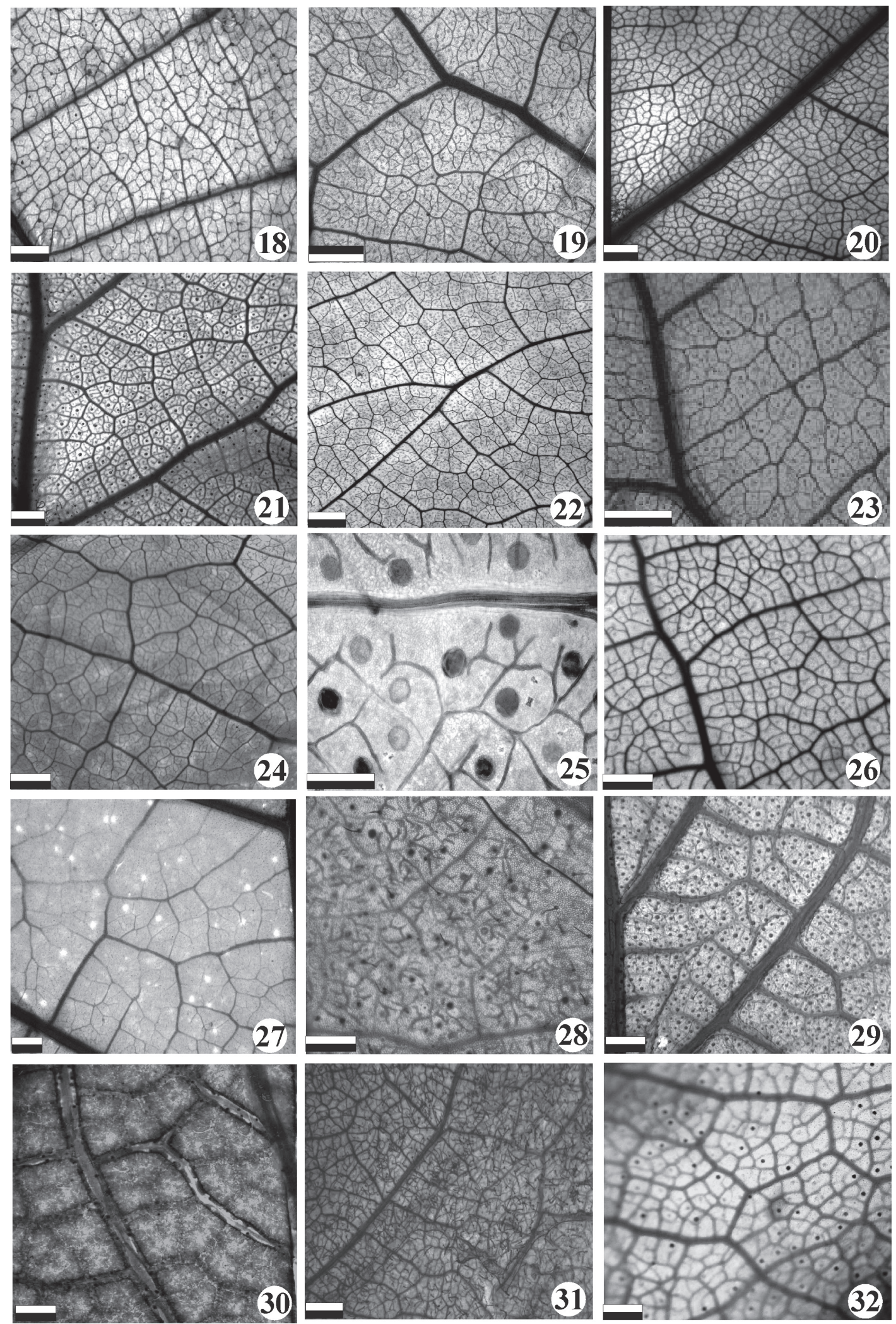

Figuras 18-32. Padrão de ramificação das nervuras nos táxons de Campomanesia Ruiz \& Pavón presentes no estado da Bahia. 18. C. anemonea Landrum. 19. C. aromatica (Aubl.) Griseb. 20. C. dichotoma (O. Berg) Mattos. 21. C. eugenioides var. desertorum (DC.) Landrum. 22. C. grandiflora (Aubl.) Sagot. 23. C. guaviroba (DC.) Kiaersk. 24. C. guazumifolia (Cambess.) O. Berg. 25. C. ilhoensis Mattos. 26. C. laurifolia Gardner. 27. C. schlechtendaliana (O. Berg) Nied. 28. C. sessiliflora var. sessiliflora (O. Berg) Mattos. 29. C. sessiliflora var. bullata (Barb. Rodr.) Landrum. 30. C. sessiliflora var. lanuginosa (Barb. Rodr. ex Chodat \& Hassl.) Landrum. 31. C. velutina (Cambess.) O. Berg. 32. C. xanthocarpa O. Berg. Escalas $=100 \mu \mathrm{m}$ (exceto Fig. 28, $50 \mu \mathrm{m})$. Pontos escuros $(23,25$, 28-29, 32) indicando glândulas laminares. Pontos translúcidos (27) indicando cavidades secretoras. 

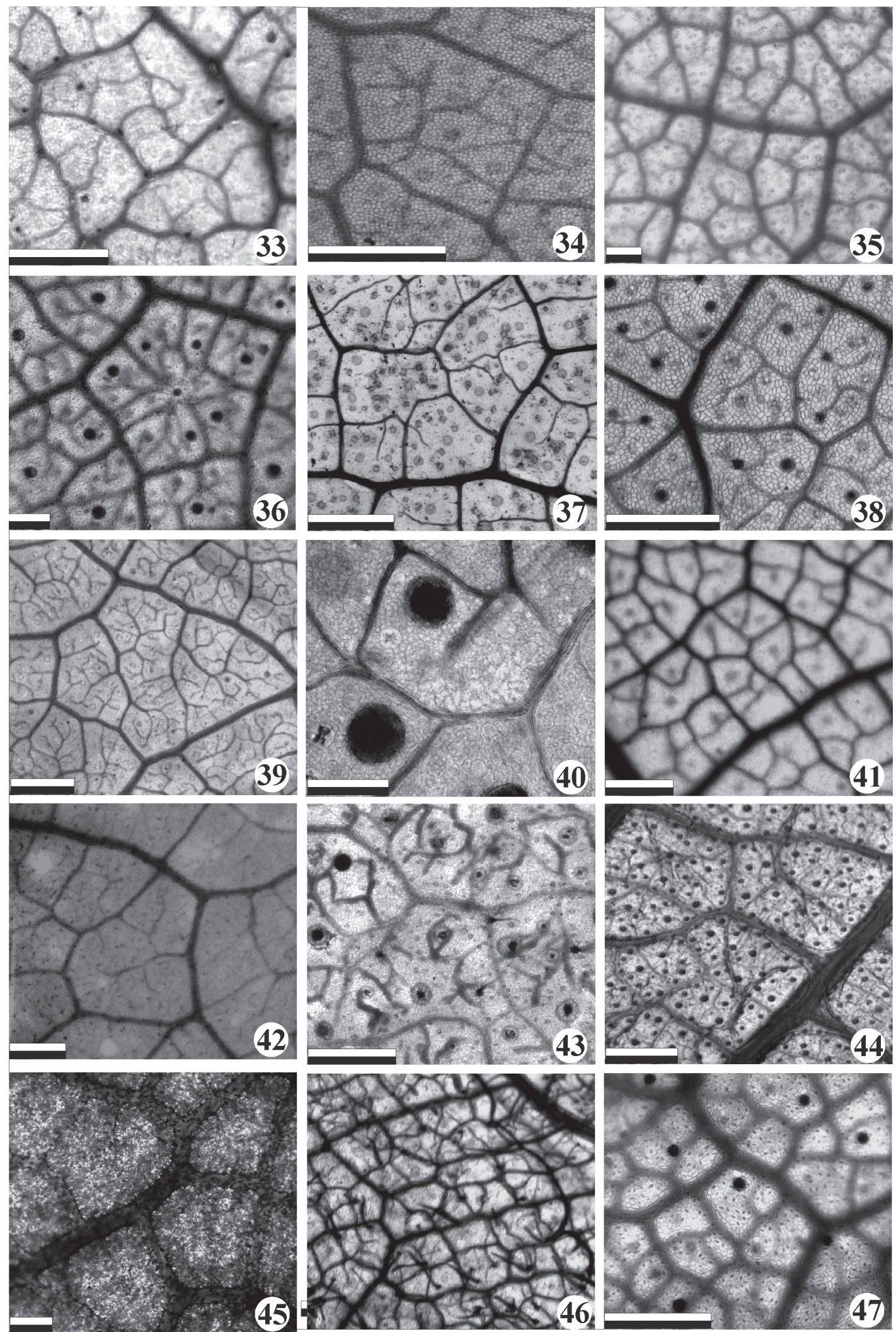

Figuras 33-47. Aréolas nos táxons de Campomanesia Ruiz \& Pavón presentes no estado da Bahia. 33. C. anemonea Landrum. 34. C. aromatica (Aubl.) Griseb. 35. C. dichotoma (O. Berg) Mattos. 36. C. eugenioides var. desertorum (DC.) Landrum. 37. C. grandiflora (Aubl.) Sagot. 38. C. guaviroba (DC.) Kiaersk. 39. C. guazumifolia (Cambess.) O. Berg. 40. C. ilhoensis Mattos. 41. C. laurifolia Gardner. 42. C. schlechtendaliana (O. Berg) Nied. 43. C. sessiliflora var. sessiliflora (O. Berg) Mattos. 44 C. sessiliflora var. bullata (Barb. Rodr.) Landrum. 45. C. sessiliflora var. lanuginosa (Barb. Rodr. ex Chodat \& Hassl.) Landrum. 46. C. velutina (Cambess.) O. Berg. 47. C. xanthocarpa O. Berg. Escalas $=50 \mu \mathrm{m}$. Pontos escuros $(33-34,36-38,40-44,47)$ indicando glândulas laminares. 

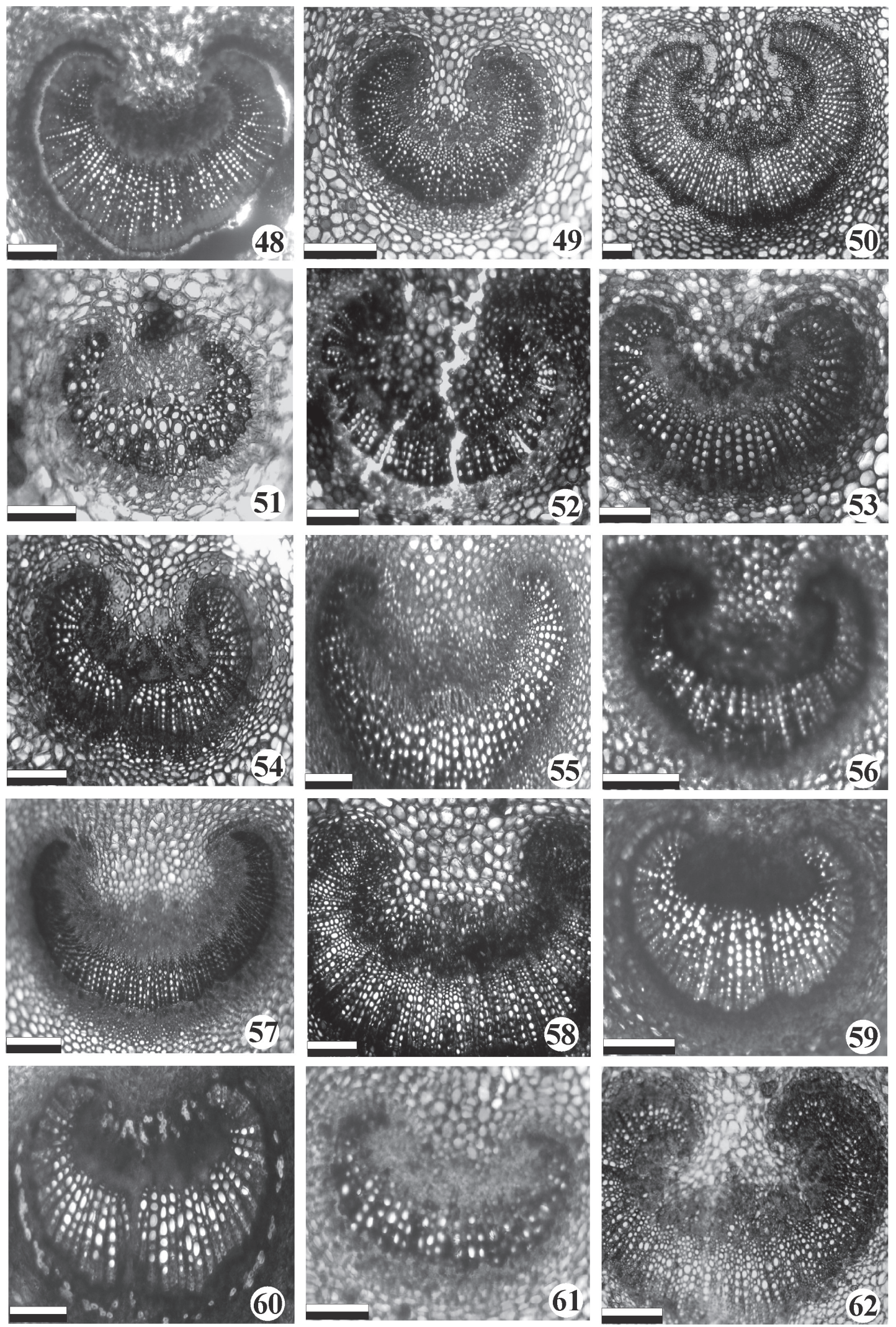

Figuras 48-62. Configuração do arco vascular no pecíolo nos táxons de Campomanesia Ruiz \& Pavón presentes no estado da Bahia. 48. C. anemonea Landrum. 49. C. aromatica (Aubl.) Griseb. 50. C. dichotoma (O. Berg) Mattos. 51. C. eugenioides var. desertorum (DC.) Landrum. 52. C. grandiflora (Aubl.) Sagot. 53. C. guaviroba (DC.) Kiaersk. 54. C. guazumifolia (Cambess.) O. Berg. 55. C. ilhoensis Mattos. 56. C. laurifolia Gardner. 57. C. schlechtendaliana (O. Berg) Nied. 58. C. sessiliflora var. sessiliflora (O. Berg) Mattos. 59. C. sessiliflora var. bullata (Barb. Rodr.) Landrum. 60. C. sessiliflora var. lanuginosa (Barb. Rodr. ex Chodat \& Hassl.) Landrum. 61. C. velutina (Cambess.) O. Berg. 62. C. xanthocarpa O. Berg. Escalas $=100 \mu \mathrm{m}$. 

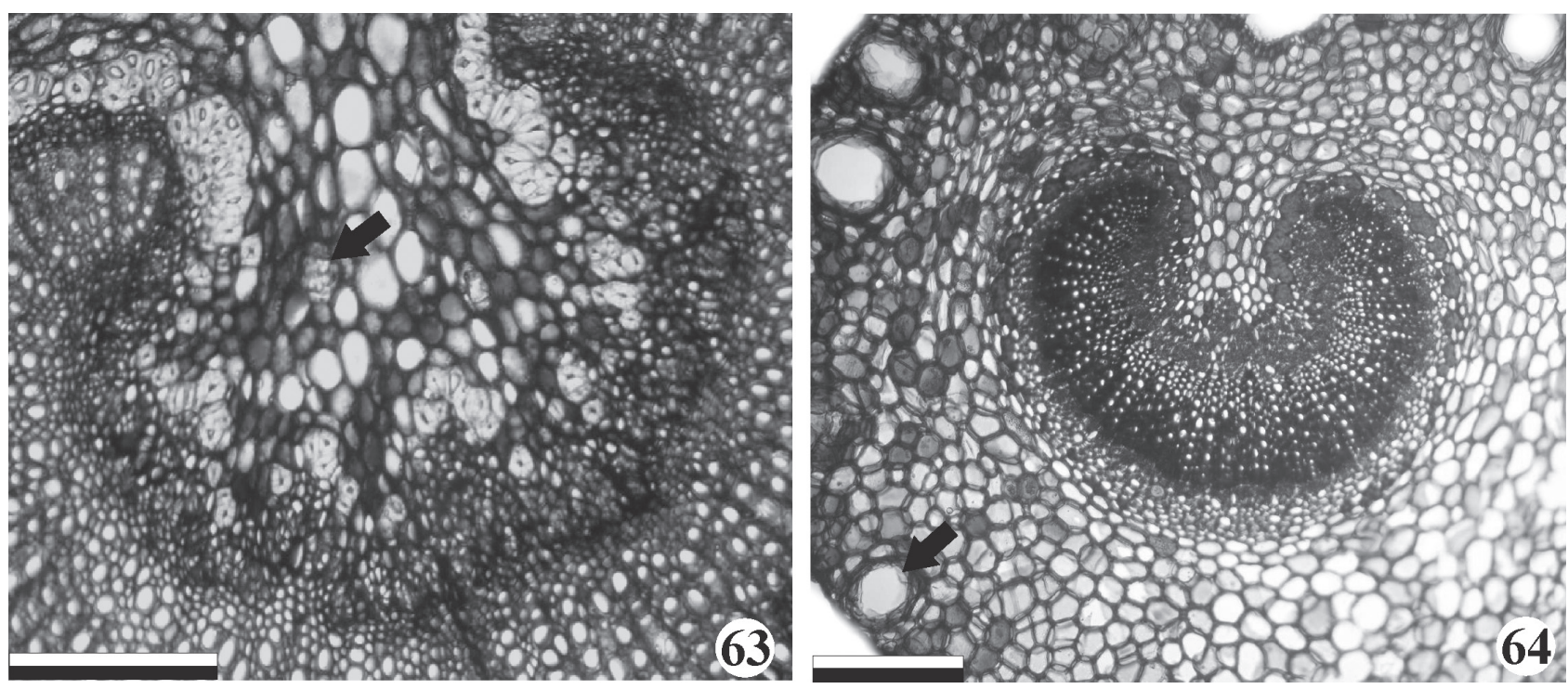

Figuras 63-64. Estruturas diversas reveladas pela secção transversal do pecíolo nos táxons de Campomanesia Ruiz \& Pavón presentes no estado da Bahia. 63. Seta indicando presença de drusas em C. dichotoma (O. Berg) Mattos. 64. Seta indicando presença de cavidades secretoras em C. aromatica (Aubl.) Griseb. Escalas = $100 \mu \mathrm{m}$.

Tabela 2. Nervação foliar nos táxons de Campomanesia Ruiz \& Pavón presentes no estado da Bahia. (+) presença. (-) ausência.

\begin{tabular}{|c|c|c|c|c|c|c|c|c|c|}
\hline \multirow[b]{2}{*}{ Táxons } & \multirow{2}{*}{$\begin{array}{c}\text { Nervuras } \\
\text { intersecundárias }\end{array}$} & \multicolumn{4}{|c|}{ Aréolas } & \multicolumn{2}{|c|}{$\begin{array}{c}\text { Natureza da bainha } \\
\text { perivascular }\end{array}$} & \multicolumn{2}{|c|}{ Configuração do arco vascular } \\
\hline & & Desenvolvimento & Arranjo & Forma & Vênulas & Mista & Parenquimática & $\begin{array}{l}\text { Extremidades } \\
\text { voltadas para o } \\
\text { centro }\end{array}$ & $\begin{array}{l}\text { Extremidades } \\
\text { fletidas }\end{array}$ \\
\hline $\begin{array}{l}\text { Campomanesia } \\
\text { anemonea }\end{array}$ & + & perfeitas & orientado & $\begin{array}{l}\text { quadrangular a } \\
\text { pentagonal }\end{array}$ & $\begin{array}{l}\text { ausentes a } \\
\text { simples }\end{array}$ & + & - & + & - \\
\hline C. aromatica & - & perfeitas & orientado & $\begin{array}{l}\text { quadrangular a } \\
\text { pentagonal }\end{array}$ & $\begin{array}{l}\text { ausentes a } \\
\text { simples }\end{array}$ & + & - & + & - \\
\hline C. dichotoma & - & perfeitas & orientado & $\begin{array}{l}\text { quadrangular a } \\
\text { pentagonal }\end{array}$ & simples & + & - & + & - \\
\hline $\begin{array}{l}\text { C. eugenioides var. } \\
\text { desertorum }\end{array}$ & + & perfeitas & orientado & $\begin{array}{l}\text { quadrangular a } \\
\text { pentagonal }\end{array}$ & ramificadas & + & - & + & - \\
\hline C. grandiflora & - & imperfeitas & ao acaso & irregular & simples & + & - & + & - \\
\hline C. guaviroba & + & imperfeitas & ao acaso & irregular & ramificadas & + & - & - & + \\
\hline C. guazumifolia & + & imperfeitas & ao acaso & irregular & ramificadas & + & - & - & + \\
\hline C. ilhoensis & + & perfeitas & orientado & $\begin{array}{l}\text { quadrangular a } \\
\text { pentagonal }\end{array}$ & $\begin{array}{l}\text { simples a } \\
\text { ramificadas }\end{array}$ & - & + & + & - \\
\hline C. laurifolia & - & $\begin{array}{l}\text { imperfeitas a } \\
\text { perfeitas }\end{array}$ & $\begin{array}{l}\text { ao acaso a } \\
\text { orientado }\end{array}$ & $\begin{array}{l}\text { irregular a } \\
\text { pentagonal }\end{array}$ & $\begin{array}{l}\text { simples a } \\
\text { ramificadas }\end{array}$ & + & - & - & + \\
\hline C. schlechtendaliana & - & imperfeitas & ao acaso & irregular & simples & + & - & + & - \\
\hline $\begin{array}{l}\text { C. sessiliflora var. } \\
\text { sessiliflora }\end{array}$ & + & imperfeitas & ao acaso & irregular & ramificadas & + & - & + & - \\
\hline $\begin{array}{l}\text { C. sessiliflora var. } \\
\text { bullata }\end{array}$ & + & perfeitas & orientado & $\begin{array}{l}\text { quadrangular a } \\
\text { pentagonal }\end{array}$ & ausentes & + & - & + & - \\
\hline $\begin{array}{l}\text { C. sessiliflora var. } \\
\text { lanuginosa }\end{array}$ & + & perfeitas & orientado & $\begin{array}{l}\text { quadrangular a } \\
\text { pentagonal }\end{array}$ & ausentes & + & - & + & - \\
\hline C. velutina & - & imperfeitas & ao acaso & $\begin{array}{l}\text { quadrangular a } \\
\text { pentagonal }\end{array}$ & $\begin{array}{l}\text { ausentes a } \\
\text { simples }\end{array}$ & - & + & + & - \\
\hline C. xanthocarpa & - & perfeitas & orientado & $\begin{array}{l}\text { quadrangular a } \\
\text { pentagonal }\end{array}$ & ausentes & + & - & - & + \\
\hline
\end{tabular}

nervura interssecundária, arco vascular com extremidades voltadas para o centro, e bainha perivascular com células parenquimáticas, podendo assim ser distinguidas de $C$. laurifolia, geralmente confundidas quando coletadas estéreis em áreas de floresta atlântica. C. anemonea foi descrita recentemente e relacionada à C. dichotoma (Landrum 2001), tendo apenas a presença de nervuras interssecundárias para diferenciá-las quando estéreis.
Estudos conduzidos em Myrtaceae (Costa et. al. 1995; Gomes \& Neves 1997; Cardoso \& Sajo 2004; Cardoso \& Sajo 2006) e outras famílias, como Apocynaceae (Rio et al. 2005) e Rubiaceae (Martínez-Cabrera et al. 2009), indicaram que os caracteres também utilizados no presente trabalho foram consistentes na separação de determinadas espécies, ainda que o mesmo não tenha acontecido para algumas Lauraceae analisadas por Moraes \& Paoli (1999) devido 
à grande variação no padrão de nervação dentro de uma mesma espécie. Para Campomanesia, podemos considerar que, embora existam semelhanças macromorfológicas entre alguns táxons, caracteres morfoanatômicos que podem ser facilmente observados, como o padrão de nervação secundário foliar e a configuração do arco vascular no pecíolo, mostraram-se úteis na identificação de espécimes coletados estéreis. Além disso, permitiram a separação dos táxons pertencentes aos grupos informais "Complexo Campomanesia guazumifolia", "Complexo C. grandiflora" e "Complexo C. xanthocarpa" citados por Landrum (1986).

\section{Agradecimentos}

Aos curadores dos herbários citados; aos amigos do Laboratório de Micromorfologia Vegetal/UEFS (LAMIV), em especial à Cláudia E. Carneiro, Kelly R.B. Leite, Ricardo L.B. de Borges e Marcos C. Dórea, pelo suporte durante a execução deste trabalho. À Fapesb (Fundação de Amparo à Pesquisa do Estado da Bahia) e ao CNPq (Conselho Nacional de Desenvolvimento Científico e Tecnológico) pelas bolsas de estudo conferidas à primeira autora durante o primeiro e segundo ano de mestrado, respectivamente.

\section{Referências Bibliográficas}

Barroso, G.M.; Peixoto, A.L.; Ichaso, C.L.F.; Costa, C.G.; Guimarães, E.F. \& Lima, H.C. 1991. Sistemática de Angiospermas do Brasil. Viçosa, UFV.

Barroso, G.M.; Amorim, M.P.; Peixoto, A.L. \& Ichaso, C.L.F. 2004. Frutos e sementes: morfologia aplicada à sistemática de dicotiledôneas. Viçosa, UFV.

Cardoso, C.M.V. \& Sajo, M.G. 2004. Vascularização foliar e a identificação de espécies de Eugenia L. (Myrtaceae) da bacia hidrográfica do Rio Tabagi, PR. Revista Brasileira de Botânica 27(1): 47-54.

Cardoso, C.M.V. \& Sajo, M.G. 2006. Nervação foliar em espécies brasileiras de Myrtaceae Adans. Acta Botanica Brasilica 20(3): 657-669.

Carr, D.J.; Carr, S.G.M. \& Lenz, J.R. 1986. Leaf venation in Eucalyptus and other genera of Myrtaceae: implications for systems of classification of venation. Australian Journal of Botany 34: 53-62.

Carrara, M.R. 1997. Estudo das espécies de Campomanesia Ruiz \& Pav. (Myrtaceae, Myrtinae) ocorrentes no estado do Rio de Janeiro. Dissertação de Mestrado, UFRJ/Museu Nacional.

Costa, C.G.; Machado, R.D. \& Fontenelle, J.B. 1995. Sistema vascular em folhas de Eugenia L. (Myrtaceae). Bradea 6(42): 345-356.

Donato, A.M. \& Morretes, B.L. 2005. Estudo anatômico das folhas de Psidium widgrenianum O. Berg (Myrtaceae), uma potencial espécie medicinal. Revista Brasileira de Farmácia 86(2): 65-70.

Donato, A.M. \& Morretes, B.L. 2007. Anatomia foliar de Eugenia brasiliensis Lam. (Myrtaceae) provenientes de áreas de restinga e de floresta. Revista Brasileira de Farmacognosia 17(3): 426-443.

Du Plessis, E. \& Van Wik, A.E. 1982. The genus Eugenia (Myrtaceae) in southern Africa: Taxometrics of foliar organography. South African Journal of Botany 1: 147-157.

Fontenelle, J.B.; Costa, C.G. \& Machado, R.D. 1994. Foliar anatomy and micromorphology of eleven species of Eugenia L. (Myrtaceae). Botanical Journal of the Linnean Society 155: 111-113.

Fontenelle, J.B.; Gomes, D.M.S. \& Machado, R.D. 1993. Anatomia foliar de Gomidesia martiana Berg e G. fenzliana Berg (Myrtaceae). Revista Brasileira de Botânica 16(1): 17-30.
Gomes, D.M.S. \& Neves, L.J. 1997. Anatomia foliar de Gomidesia spectabilis (DC.) Berg. e Gomidesia nitida (Vell.) Legr. (Myrtaceae). Rodriguésia 45/49(71/75): 51-70.

Gomes, S.M.; Somavilla, N.S.D.N.; Gomes-Bezerra, K.M.; Miranda, S.C.; De-Carvalho, P.S. \& Graciano-Ribeiro, D. 2009. Anatomia foliar de espécies de Myrtaceae: contribuições à taxonomia e filogenia. Acta Botanica Brasilica 23(1): 223-238.

Govaerts, R.; Sobral, M.; Ashton, P.; Barrie, F.; Holst, B.K.; Landrum, L.R.; Matsumoto, K.; Mazine, F.F.; Nic Lughadha, E.; Proença, C.E.B.; Silva, L.H.S.; Wilson, P.G. \& Lucas, E.J. 2008. World Checklist of Myrtaceae. Kew, Royal Botanic Garden.

Hickey, L.J. 1973. Classification of the architecture of Dicotyledonous leaves. American Journal of Botany 60(1): 17-33.

Hickey, L.J. 1979. A revised classification of the architecture of Dicotyledonous leaves. Pp. 23-39. In: Metcalfe, C.R. \& Chalk, L. Anatomy of the Dicotyledons - Systematics anatomy of leaf, and stem, with a brief history of the subject. $2^{\text {nd }}$ ed. Oxford, Clarendon Press.

Holmgren, P.K.; Holmgren, N.H. \& Barnett, L.C. 1990. Index Herbariorum - Part I: The Herbaria of the World. New York, The New York Botanic Garden Press.

Howard, R.A. 1979. The petiole. Pp. 88-96. In: Metcalfe, C.R. \& Chalk, L. Anatomy of the Dicotyledons - Systematics anatomy of leaf, and stem, with a brief history of the subject. $2^{\text {nd }}$ ed. Oxford, Clarendon Press.

Kawasaki, M.L. \& Landrum, L.R. 1997. A rare and potentially economic fruit of Brazil: cambuci, Campomanesia phaea (Myrtaceae). Economic Botany 51(4): 403-407.

Klucking, E.P. 1988. Leaf venation patterns - Myrtaceae. Berlin, J.Cramer.

Kraus, J.E. \& Arduin, M. 1997. Manual básico de métodos em morfologia vegetal. Seropédica, EDUR.

Landrum, L.R. 2001. Two new species of Campomanesia (Myrtaceae) from Espírito Santo and Bahia, Brazil. Brittonia 53(4): 534-538.

Landrum, L.R. \& Kawasaki, M.L. 1997. The genera of Myrtaceae in Brazil: an illustrated synoptic treatment and identification keys. Brittonia 49(4): 508-536.

Landrum, L.R. \& Oliveira, M.I.U. 2010. A new species of Campomanesia from Bahia, Brazil, based on specimens collected by J.S. Blanchet over 150 years ago. Journal of the Botanical Research Institute of Texas 4(2): 603-607.

Landrum, L.R. 1986. Campomanesia, Pimenta, Blepharocalyx, Legrandia, Acca, Myrrhinium and Luma (Myrtaceae). Flora Neotropica - Monograph 45: 7-72.

Legrand, C.D. \& Klein, R.M. 1977. Mirtáceas - Campomanesia, Feijoa, Britoa, Myrrhinium, Hexaclamys, Siphoneugena, Myrcianthes, Neomitranthes e Psidium. Pp. 573-634. In: Reitz, R. (Ed.). Flora Ilustrada Catarinense. Itajaí, CNPq/IBDF/HBR.

Lorenzi, H.; Sartori, S.F.; Bacher, L.B. \& Lacerda, M. 2006. Frutas brasileiras e exóticas cultivadas (de consumo in natura). Nova Odessa, Instituto Plantarum de Estudos da Flora.

Lucas, E.J.; Belsham, S.R.; Nic Lughadha, E.M.; Orlovich, D.A.; Sakuragui, C.M.; Chase, M.W. \& Wilson, P.G. 2005. Phylogenetic patterns in the fleshy-fruited Myrtaceae - preliminary molecular evidence. Plant Systematic and Evolution 251: 35-51.

Lucas, E.J.; Harris, S.A.; Mazine, F.F.; Belsham, S.R.; Nic Lughadha, E.M.; Telford, D.A.; Gasson, P.E. \& Chase, M.W. 2007. Suprageneric phylogenetics of Myrteae, the generically richest tribe in Myrtaceae (Myrtales). Taxon 56(4): 1105-1128.

Martínez-Cabrera, D.; Terrazas, T. \& Ochoterena, H. 2009. Foliar and petiole anatomy of Tribe Hamelieae and other Rubiaceae. Annals of the Missouri Botanical Garden 96: 133-145.

McVaugh, R. 1968. The genera of American Myrtaceae - an interim report. Taxon 17: 354-418.

Metcalfe, C.R. \& Chalk, L. 1950. Anatomy of the Dicotyledons - Leaves, stem, and wood in relation to taxonomy with notes on economic uses. Oxford, Clarendon Press.

Moraes, P.L.R \& Paoli, A.A.S. 1999. Epiderme e padrão de venação foliar de espécies de Lauraceae. Acta Botanica Brasilica 13(1): 87-97.

Oliveira, M.I.U.; Funch, L.S. \& Landrum, L.R. no prelo. Flora da Bahia Myrtaceae: Campomanesia Ruiz \& Pavón. Sitientibus, Série Ciências Biológicas. 
Proença, C.E.B.; Jennings, L.V. \& Lucas, E.J. 2011. Two new species of Myrtaceae (Myrteae) from northern South America. Brittonia 63: 46-50.

Proença, C.E.B.; Soares-Silva, L.H.; Silva, P.I.T. \& Fank-de-Carvalho, S.M. 2010. Two new endemic species of Myrtaceae and na anatomical novelty from the Highlands of Brazil. Kew Bulletin 65: 1-6.

Rio, M.C.S.; Kinoshita, L.S. \& Castro, M.M. 2005. Anatomia foliar como subsídio para a taxonomia de espécies de Forsteronia G.Mey. (Apocynaceae) dos cerrados paulistas. Revista Brasileira de Botânica 28(4): 713-726.
Shobe, W.R. \& Lersten, N.R. 1967. A technique for clearing and staining Gymnosperm leaves. Botanical Gazette 128(2): 150-152.

Wilson, P.G.; O’Brien, M.M.; Gadek, P.A. \& Quinn, C.J. 2001. Myrtaceae revisited: a reassessment of infrafamiliar groups. American Journal of Botany 88(11): 2013-2025.

Wilson, P.G.; O’Brien, M.M.; Heslewood, M.M. \& Quinn, C.J. 2005. Relationships within Myrtaceae sensu lato based on a matk phylogeny. Plant Systematic and Evolution 251: 3-19. 